\title{
Charge order, superconductivity, and a global phase diagram of doped antiferromagnets
}

\author{
Matthias Vojta and Subir Sachdev \\ Department of Physics, Yale University \\ P.O. Box 208120, New Haven, CT 06520-8120, USA
}

(November 8, 1999)

\begin{abstract}
We investigate the interplay between lattice-symmetry breaking and superconducting order in a twodimensional model of doped antiferromagnets, with long-range Coulomb interactions and $\operatorname{Sp}(2 N)$ spin symmetry, in the large- $N$ limit. Our results motivate the outline of a global phase diagram for the cuprate superconductors. We describe the quantum transitions between the phases, the evolution of their fermion excitation spectrum, and the experimental implications.
\end{abstract}

A number of recent experiments have found a fich variety of phases in the cuprate superconductors 1 . The various ground states can be distinguished by the manner in which they preserve, or spontaneously break, three distinct and familiar symmetries of the Hamiltonian: (a) the electromagnetic $U(1)$ symmetry, $\mathcal{S}$, which is broken in the $d$-wave superconducting phase, but is preserved in an insulating ground state; (b) the $\mathrm{SU}(2)$ spin rotation symmetry, $\mathcal{M}$, which is broken in magnetically ordered phases; and (c) the symmetry of square lattice translations and rotations, $\mathcal{C}$, which we will consider broken if an observable invariant under $\mathcal{S}$ and $\mathcal{M}$, like the charge density, is not identical on every site and every bond. We shall take the point of view here that all the phases are conventionally characterized by the manner in which $\mathcal{S}, \mathcal{C}$, and $\mathcal{M}$ are broken, and have no 'exotic' properties or excitations, i.e., in principle, an appropriate electron Hartree-Fock/RPA/BCS theory, with perturbative corrections, can be found; the anomalous finite temperature $(T)$ properties are then believed to be signatures of quantum-critical points separating these phasest 0 .

This paper will describe the $T=0$, global phase diagram of two-dimensional, doped antiferromagnets by discussing the competition between phases in which one or more of the $\mathcal{S}, \mathcal{C}$, and $\mathcal{M}$ symmetries may be broken. Among our results will be the complete quantitative solution of a microscopic model of a doped antiferromagnet for the case where the $\mathcal{M}$ symmetry is generalized 1 from $\mathrm{SU}(2)$ to $\mathrm{Sp}(2 N)$, (note $\mathrm{SU}(2) \cong \mathrm{Sp}(2))$ and the large- $N$ limit is taken under a particular representation of $\operatorname{Sp}(N)$. The simplifying feature of this limit is that it restricts attention to the portion of the phase diagram (see Fig. 11 below) where the $\mathcal{M}$ symmetry remains unbroken; however, it does allow a realistic description of the subtle and complicated interplay between the $\mathcal{C}$ and $\mathcal{S}$ symmetries. Our results include $(i)$ computation of the doping dependence of the charge-ordering configuration and the evolution of the ordering wavevector, (ii) computation of the single-particle fermion spectrum, measurable in photoemission experiments, in phases with $\mathcal{C}$ and $\mathcal{S}$ broken, and (iii) proposal of a quantum-critical field-theoretic model to explain the recently observed 1 anomalous $T$ and frequency dependence of the photoemission line-width.
We will consider the following extended " $t-J$ " Hamiltonian for fermions, $c_{i \alpha}$, on the sites, $i$, of a square lattice with spin $\alpha=1 \ldots 2 N(N=1$ is the physical value):

$$
\begin{array}{r}
\mathcal{H}=\sum_{i>j}\left[-\frac{t_{i j}}{N} c_{i \alpha}^{\dagger} c_{j \alpha}+\text { H.c. }+\frac{V_{i j}}{N} n_{i} n_{j}\right. \\
\left.+\frac{J_{i j}}{N}\left(\mathbf{S}_{i} \cdot \mathbf{S}_{j}-\frac{n_{i} n_{j}}{4 N}\right)\right] .
\end{array}
$$

Here $n_{i}=c_{i \alpha}^{\dagger} c_{i \alpha}$ is the on-site charge density, and the spin operators $\mathbf{S}_{i}$ are fermion bilinears times the traceless generators of $\operatorname{Sp}(2 N)$. We will be primarily concerned with the case where the fermion hopping, $t_{i j}$, and exchange, $J_{i j}$, act only when $i, j$ are nearest neighbors, in which case $t_{i j}=t$ and $J_{i j}=J$; however, we will occasionally refer to cases with second neighbor hopping $\left(t^{\prime}\right)$ or exchange $\left(J^{\prime}\right)$. The Coulomb interaction between the electrons is represented by the on-site constraint $n_{i} \leq N$, and the off-site repulsive interactions $V_{i j}$ which fall off as the inverse separation between the sites. The $V_{i j}$ are included to counter-act the phase separation tendency of the $t$ - $J$ model10 12 , and play a key role in our analysis. We shall be interested in describing the ground state of $\mathcal{H}$ as a function of its couplings and the average doping concentration, $\delta$, which is fixed by $\left(1 / N_{s}\right) \sum_{i}\left\langle n_{i}\right\rangle=N(1-\delta)$, where $N_{s}$ is the (infinite) number of sites.

The proposed phase diagram of $\mathcal{H}$ is shown in Fig. 1. First, consider the vertical line, $\delta=0$. Below $X$, magnetic Néel order is present and so $\mathcal{M}$ is broken; however, the charge densities are identical on every bond and site, and so $\mathcal{C}$ is preserved, as is $\mathcal{S}$ because the ground state is an insulator. Above $X$, there is a transition to a quantum paramagnet and $\mathcal{M}$ symmetry is restored; this transition was studied in Refs. 13,8, and it was argued that $\mathcal{C}$ was necessarily broken in the quantum paramagnet leading to spin-Peierls order. We can also view the spin-Peierls order as bond-centered charge-density wave with a $2 \times 1$ unit cell14. Recent work 15 has shown strong evidence for this order in the $N=1$ model with $J^{\prime}>0$.

We now describe the evolution of the ground state with increasing $\delta$ along $A_{1}$. The large- $N$ limit is taken, as described earlier 11 , by minimizing the saddle point free 


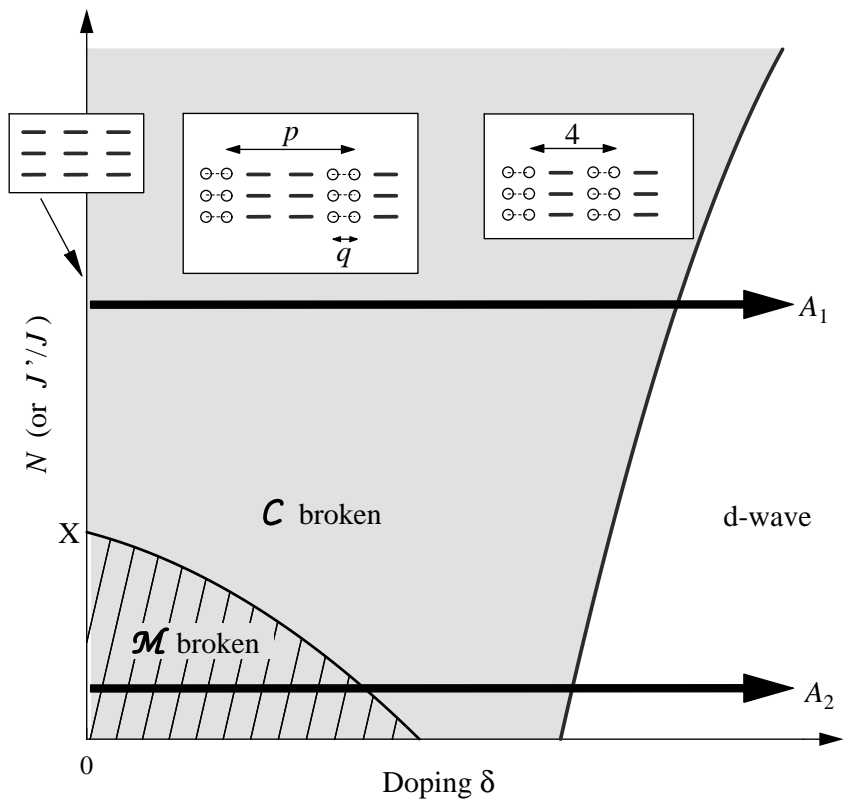

FIG. 1. Schematic, proposed, ground state phase diagram of $\mathcal{H}$ as a function of the doping $\delta$ for physically reasonable values of $t, J$ and $V$. The vertical axis represents a parameter which measures the strength of quantum spin fluctuations - it increases linearly with $N$ but can also be tuned continuously by $J^{\prime} / J$. The magnetic $\mathcal{M}$ symmetry is broken in the hatched region, while $\mathcal{C}$ symmetry is broken (with accompanying charge-density modulation) in the shaded region; there are numerous additional phase transitions at which the detailed nature of the $\mathcal{M}$ or $\mathcal{C}$ symmetry breaking changes these are not shown. For $\delta=0, \mathcal{M}$ symmetry is broken only below the critical point $X$, while $\mathcal{C}$ symmetry is broken only above $X$. The superconducting $\mathcal{S}$ symmetry is broken for all $\delta>0$ at large $N$; for smaller $N$, the $\mathcal{S}$ can be restored at small $\delta$ by additional $\mathcal{C}$ breaking along the vertical axis for the states in the inset-this is not shown. The superconductivity is pure $d$-wave only in the large $\delta$ region were $\mathcal{C}$ and $\mathcal{M}$ are not broken. The arrow $A_{1}$ represents the path along which quantitative results are obtained in this paper, while $A_{2}$ is the experimental path. The nature of the $\mathcal{C}$ symmetry breaking along path $A_{1}$ is also sketched: the thick and dashed lines indicate varying values of $\left|Q_{i j}\right|$ (proportional to the bond charge density) on the links, while the circles represent $b_{i}^{2}$ (proportional to the site hole density). The charge densities on the links and sites not shown take values consistent with the symmetries of the figures shown. We expect that the nature of the $\mathcal{C}$ symmetry breaking will not change significantly as we move from $A_{1}$ to $A_{2}$, and across the phase boundary where $\mathcal{M}$ is broken: this suggests the appearance of collinearly polarized spin-density waves, which break both $\mathcal{C}$ and $\mathcal{M}$, and hich undergo an 'anti-phase' shift across the hole-rich stripes 16 . energy with respect to the site charge density $N(1-$ $\left.b_{i}^{2}\right)=\left\langle n_{i}\right\rangle$ and the complex bond pairing amplitude $N Q_{i j}=\left\langle\mathcal{J}^{\alpha \beta} c_{i \alpha}^{\dagger} c_{j \beta}^{\dagger}\right\rangle /\left(b_{i} b_{j}\right)$ (where $b_{i}^{2}$ is the hole density at site $i$ and $\mathcal{J}$ denotes the $\operatorname{Sp}(2 N)$-invariant antisymmetric tensor), while maintaining certain local and global constraints. There have been a number of related earlier mean-field studies 17 , but they have all (with the exception of Ref. 11) restricted attention to the case where $b_{i}$ and $\left|Q_{i j}\right|$ are spatially uniform (note that $\left|Q_{i j}\right|$ has the same symmetry signature as the bond charge density, and is therefore a measure of its value). However such solutions are usually unstable, and at best metastable, at low doping; here we have attempted to find the true global minima of the saddle-point equations, while allowing for arbitrary spatial dependence: such a procedure leads to considerable physical insight, and also leads to solutions in accord with recent experimental observations.

First, at $\delta=0$ along $A_{1}$ we find the fully dimerized, insulating spin-Peierls (or $2 \times 1$ bond charge-density wave) solution 18 in which $\left|Q_{i j}\right|$ is non-zero only on the bonds shown in Fig. 1. Moving to small non-zero $\delta$ along $A_{1}$, our numerical search always yielded lowest energy states with $\mathcal{C}$ hroken, consisting of bond-centered charge-density waves 19 with a $p \times 1$ unit cell, as shown in Fig. 11. We always found $p$ to be an even integer, reflecting the dimerization tendency of the $\delta=0$ solution. Within each $p \times 1$ unit cell, we find that the holes are concentrated on a $q \times 1$ region, with a total linear hole density of $\rho_{\ell}$. A key property is that $q$ and $\rho_{\ell}$ remain finite, while $p \rightarrow \infty$, as $\delta \rightarrow 0$. Indeed, the values of $q$ and $\rho_{\ell}$ are determined primarily by $t, J$, and the nearest-neighbor value of $V_{i j}=V_{n n}$, and are insensitive to $\delta$ and longer range parts of $V_{i j}$. For $\delta \rightarrow 0$, we found that $q=2$ was optimum for a wide range of parameter values, while larger values of $q(q \geq 4)$ appear for smaller values of $V_{n n}$; specifically we had $q=2, \rho_{\ell}=0.42$ at $t / J=1.25, V_{n n} / t=0.6$, and $q=4, \rho_{\ell}=0.8$ at $t / J=1.25, V_{n n} / t=0.5$. The limit $V_{n n} \rightarrow 0$ leads to $q \rightarrow \infty$ which reflects the tendency to phase separation in the "bare" $t-J$ model. The evolution of $p$ with $\delta$ is shown in Fig. 2. Note that there is a large plateau at $p=4$ around doping $\delta=1 / 8$, and, for some parameter regimes, this is the last state before $\mathcal{C}$ is restored at large $\delta$; indeed $p=4$ is the smallest value of $p$ for which our mean-field theory has solutions with $b_{i}$ not spatially uniform. Experimentally 1 , 2 , a pinning of the charge order at a wavevector $K=1 / 4$ is observed, and we consider it significant that this value emerges naturally from our theory.

Our large- $N$ theory only found states in which the ordering wavevector $K$ was quantized at the rational plateaus in Fig. 2. However, for smaller $N$ we expect that irrational, incommensurate, values of $K$ will appear, and interpolate smoothly between the plateau regions.

In our large- $N$ theory, each $q$-width stripe above is a one-dimensional superconductor, while the intervening $(q-p)$-width regions are insulating. However, fluctuation corrections will couple with superconducting regions, 


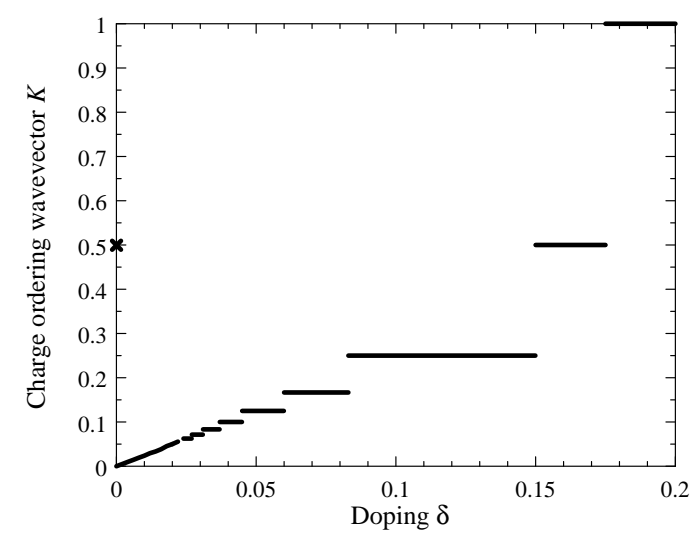

FIG. 2. The charge-ordering wavevector, $K$, (in reciprocal lattice units) as a function of $\delta$ at $N=\infty$ for $t / J=1.25$, $V_{n n} / t=0.6$ (where $q=2$ ). For the states in Fig. 1. $K=1 / p$. We have $K=1 / 2$ at $\delta=0$. The $K=1$ value at large $\delta$ has $\mathcal{C}$ symmetry restored, and is a pure $d$-wave superconductor. For other values of parameters, the $K=1 / 2$ plateau does not occur, and there is a direct jump from $K=1$ to $K=1 / 4$ (or smaller) (see Fig. B).

yielding an effective theory discussed in Section VII of Ref. 20 with their dimensionless parameter $K \sim N$. This implies that Josephson pair tunneling between the onedimensional superconductors is a relevant perturbation at sufficiently large $N$, leading to two-dimensional superconductivity. However, the bare pair-tunneling amplitude is exponentially small in $p$, while the Coulomb interaction between the hole-rich regions falls off only as $1 / p$ - the latter can then dominate for smaller $N$ and $\delta$, leading to further $\mathcal{C}$ breaking along the vertical stripe directions, and a transition to a two-dimensional insulating state with $\mathcal{S}$ restored and an even number of electrons per unit cell. Such an insulating state is more likely at rational $\delta$, when the charge-ordering period along the vertical stripe direction is commensurate with the lattice.

We show a fixed $\delta=1 / 8$, large $N$, cross-section of our results in Fig. 3. The transition from a $d$-wave superconductor, with $\mathcal{C}$ unbroken, to the fully-formed $p \times 1$ stripes discussed above can either be first-order, or via intermediate states with partial stripe order. In the latter case, there is first a continuous transition to a state with $\mathcal{C}$ symmetry breaking at $p=2$ - every site is equivalent in such a state, and so the site charge density is uniform while there is a modulation in the bond charge density; this state can also be viewed as possessing coexisting superconducting and spin-Peierls order 11 . To our knowledge a $p=2$ charge-ordered superconducting state has not been experimentally detected, but a search for one should be worthwhile. There is a second secondorder transition to $p=4$ state with partial stripe order, before the fully-formed $p=4, q=2$ state with intervening insulating stripes appears (Fig. 3). Larger values of $V$ suppress phases with a non-uniform distribution of site charge densities; such phases also disappear in the limits of small $t / J$, and $t / J \rightarrow \infty$.

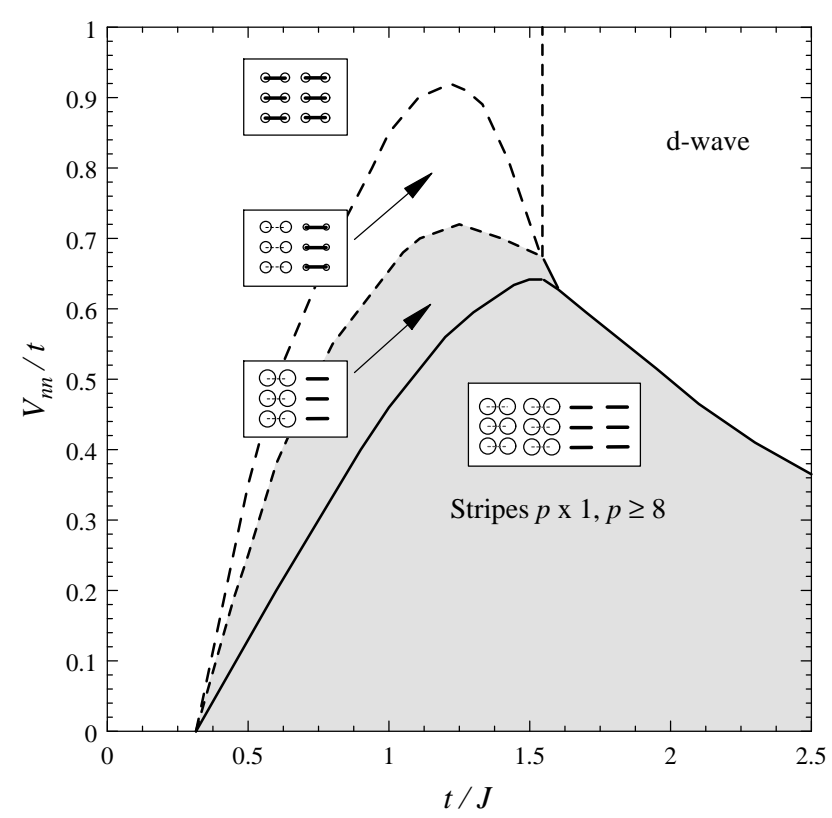

FIG. 3. Ground states of $\mathcal{H}$ at $\delta=1 / 8$ and $N=\infty$. Full (dashed) lines indicate first- (second-) order transitions. All states have superconducting order, but the superconductivity is one-dimensional (only at $N=\infty$ ) in the phases with full stripe order (shaded).

We now discuss the fermion excitation spectrum in the states found above. The $d$-wave superconductor of course has gapless, linearly dispersing fermion excitations along the $(1, \pm 1)$ directions in the Brillouin zone. The various charge-ordered phases in general show a gapped spectrum, see Fig. 4. In the fully striped phases the fermion energy is independent of $k_{x}$ (the momentum perpendicular to the stripes), the dispersion minimum is near $(0, \pm 1 / 4)$. In the $p=2$ phases (and also for $p=4$ and partial stripe order) the minimum of the energy is at complex values of pairing amplitudes $Q_{i j}$; these states break time-reversal symmetry $\mathcal{T}$ and their fermionic excitations are fully gapped. However, if we restrict our attention to states without $\mathcal{T}$-breaking, then upon decreasing $t / J$ in the $d$-wave superconductor (at large $V$, see Fig. 3) the gapless fermions survive across the $\mathcal{C}$-breaking transition to the $p=2$ phase; the excitation gap then opens at smaller $t / J$ (i.e. at a finite dimerization).

Finally, we describe the critical behavior near the quantum transitions. Consider first, the initial onset of $\mathcal{C}$-breaking from the $d$-wave superconductor (Fig. 11). For large $N$, this transition, if second-order, occurs at the wavevector $(K=1 / 2,0)$ which does not equal the separation between any two gapless Fermi points; so the charge order parameter does not couple efficiently to the fermionic excitations. In this case, the effective quantum critical theory contains only two real scalars $\left(\phi_{x}\right.$, $\left.\phi_{y}\right)$, describing the ordering along the two axes, and has a three spacetime dimensional, 'relativistically' invariant action with the symmetry of the $Z_{4}$ clock model. For 


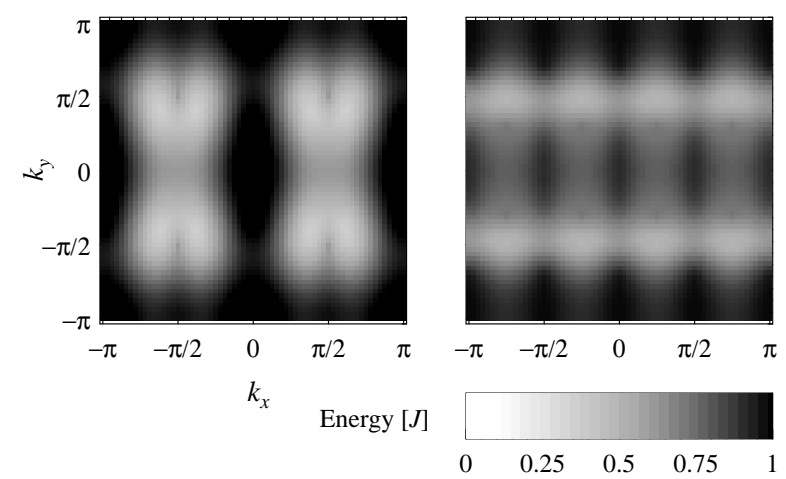

FIG. 4. Dispersion of the fermionic excitation spectrum obtained from the mean-field solution of $\mathcal{H}$ at $\delta=1 / 8$. Left: $p=2$ phase at $t / J=1.5, V / t=1$, i.e., very close to the transition to the $d$-wave phase. Right: $p=4$ with partial stripe order at $t / J=1.25, V / t=0.8$. Both spectra are fully gapped. Here, $k_{x}$ is the momentum parallel to the charge-ordering wavevector (i.e., the stripes run in $y$ direction).

smaller $N$, we consider it likely that the initial $\mathcal{C}$ breaking will occur at a wavevector $(K, 0)$, which is incommensurate with the underlying lattice, but which does exactly equal the separation between gapless Fermi points in the superconductor. The critical quantum field theory will now contain two complex scalars $\left(\left(\Phi_{x}, \Phi_{y}\right)\right.$-their phases represent the ability to freely slide the charge-density wave with respect to the lattice) coupled to the four 'Dirac' fermions of the $d$-wave superconductor. Its effective action has the form $S=S_{f}+S_{\Phi}+S_{\lambda} ; S_{f}$ is the fermion bilinear of the $d$-wave superconductor containing gapless Fermi points at $(K / 2, K / 2),(-K / 2, K / 2)$, $(-K / 2,-K / 2)$ and $(K / 2,-K / 2)$, and we will denote the components of $c_{i \alpha}$ in the vicinity of these points by $f_{a \alpha}$ respectively $(a=1 \ldots 4) ; S_{\Phi}$ contains second order spatial and time derivatives of $\Phi_{x, y}$ and polynomial interaction terms, all invariant under the uniform phase change $\Phi_{x, y} \rightarrow e^{i \theta_{x, y}} \Phi_{x, y}$ and under $\Phi_{x} \leftrightarrow \Phi_{y} ; S_{\lambda}$ couples the $f_{a \alpha}$ and $\Phi_{x, y}$, and the symmetries allow the following two independent terms, free of gradients:

$$
\begin{aligned}
& \lambda_{1} \mathcal{J}^{\alpha \beta}\left(\Phi_{x} f_{1 \alpha} f_{4 \beta}+\Phi_{x}^{*} f_{2 \alpha} f_{3 \beta}+\Phi_{y} f_{2 \alpha} f_{1 \beta}+\Phi_{y}^{*} f_{3 \alpha} f_{4 \beta}\right) \\
& +\lambda_{2}\left(\Phi_{x} f_{2 \alpha}^{\dagger} f_{1 \alpha}+\Phi_{x} f_{3 \alpha}^{\dagger} f_{4 \alpha}+\Phi_{y} f_{4 \alpha}^{\dagger} f_{1 \alpha}+\Phi_{y} f_{3 \alpha}^{\dagger} f_{2 \alpha}\right)
\end{aligned}
$$

and their Hermitian conjugates. We propose that it is this quantum field theory, describing the $T=0$ transition at which $\left\langle\Phi_{x, y}\right\rangle$ become non-zero in the presence of superconductivity, whose $T>0$ correlators describe the observed quantum-critica scaling of the fermion momentum distribution functione. Direct observation of charge fluctuations at wavevectors $(K, 0),(0, K)$, with $K$ consistent with photoemission, will be a test of this scenario.

We turn to the quantum transition where $\mathcal{M}$ symmetry is broken which is located at lower $\delta$. Assuming that this is at a point where the fermion spectrum is already fully gapped, or the separation between any gapless Fermi points is not equal to the spin ordering wavevector, we can conclude that this transition is described by the relativistic quantum $O(3)$ non-linear sigma model (for $N=1$ ). Such a scenario provides a natural explanation for the crossovers in NMR experiments 3 .

This paper has used quantitative calculations of a microscopic model in a large- $N$ limit to motivate a scenario in which superconducting, spin- and charge-density wave instabilities compete as the system evolves from an insulating antiferromagnet to a $d$-wave superconductor. Many aspects are consistent with recent experiments, and more stringent tests should be possible in the future.

We thank V. Emery, M. P. A. Fisher, D. Scalapino and S. White for useful discussions. This research was supported by US NSF Grant No DMR 96-23181 and by the DFG (VO 794/1-1).

${ }^{1}$ J. M. Tranquada, J. Phys. Chem. Solids 59, 2150 (1998).

${ }^{2}$ S. Wakimoto et al., Phys. Rev. B 60, R769 (1999); Y. S. Lee et al., ibid 60, 3643 (1999).

${ }^{3}$ A. W. Hunt et al., Phys. Rev. Lett. 82, 4300 (1999); T. Imai et al., ibid 70, 1002 (1993) and 71, 1254 (1993); S. Fujiyama et al., Phys. Rev. Lett. 83, 2014 (1999).

${ }^{4}$ S. Sachdev and J. Ye, Phys. Rev. Lett. 69, 2411 (1992).

${ }^{5}$ C. Castellani et al., Phys. Rev. Lett. 75, 4650 (1995).

${ }^{6}$ S.-C. Zhang, Science 275, 1089 (1997).

${ }^{7}$ R. B. Laughlin, Adv. in Phys. 47, 943 (1998).

${ }^{8}$ N. Read and S. Sachdev, Phys. Rev. Lett. 66, 1773 (1991).

${ }^{9}$ T. Valla et al., Science 285, 2110 (1999).

${ }^{10}$ V. J. Emery, S. A. Kivelson, and H. Q. Lin, Phys. Rev. Lett. 64, 475 (1990); Phys. Rev. B 42, 6523 (1990).

11 S. Sachdev and N. Read, Int. J. Mod. Phys. B 5, 219 (1991).

12 C. Hellberg and E. Manousakis, Phys. Rev. Lett. 78, 4069 (1997).

13 N. Read and S. Sachdev, Phys. Rev. Lett. 62, 1694 (1989); Phys. Rev. B 42, 4568 (1990).

${ }^{14}$ Strictly speaking, the bond charge density modulation is not present in the insulating spin model, but it is present in the parent Hubbard-like model. The interpretation of a spin-Peierls state as a bond-charge-density-wave state also appears in M. Fabrizio et al., Phys. Rev. Lett. 83, 2014 (1999).

${ }^{15}$ V. N. Kotov et al., cond-mat/9903154; R. R. P. Singh et al., cond-mat/9904064 V. N. Kotov and O. P. Sushkov, cond-mat/9907178; O. P. Sushkov, cond-mat/9907400.

16 J. Zaanen and O. Gunnarsson, Phys. Rev. B 40, 7391 (1989); H. Schulz, J. de Physique 50, 2833 (1989).

17 G. Baskaran et al, Solid State Commun. 63, 973 (1987); A. E. Ruckenstein et al, Phys. Rev. B 36, 857 (1987); B. G. Kotliar and J. Liu, Phys. Rev. B 38, 5142 (1988).

18 N. Read and S. Sachdev, Nucl. Phys. B 316, 609 (1989); D. Rokhsar, Phys. Rev. B 42, 2526 (1990).

19 S. R. White and D. J. Scalapino, Phys. Rev. Lett. 80, 1272 (1998); 81, 3227 (1998); Phys. Rev. B 60, R753 (1999).

${ }^{20}$ H.-H. Lin et al., Phys. Rev. B 56, 6569 (1997). 